\title{
Diffusion Tensor Magnetic Resonance Imaging of Wallerian Degeneration in Rat Spinal Cord after Dorsal Root Axotomy
}

\author{
Jiangyang Zhang, ${ }^{1}$ Melina Jones, ${ }^{2}$ Cynthia A. DeBoy, ${ }^{2}$ Daniel S. Reich, ${ }^{1,2}$ Jonathan A. D. Farrell, ${ }^{1,3,7}$ Paul N. Hoffman, ${ }^{2,4}$ \\ John W. Griffin, ${ }^{2}$ Kazim A. Sheikh, ${ }^{2}$ Michael I. Miller, ${ }^{5,6}$ Susumu Mori, ${ }^{1,7}$ and Peter A. Calabresi ${ }^{2}$ \\ ${ }^{1}$ Russel H. Morgan Department of Radiology and Radiological Science, Departments of ${ }^{2}$ Neurology, ${ }^{3}$ Biophysics and Biophysical Chemistry, \\ ${ }^{4}$ Ophthalmology, and ${ }^{5}$ Biomedical Engineering, Johns Hopkins University School of Medicine, Baltimore, Maryland 21205 , ${ }^{6}$ Center of Imaging Science, \\ Johns Hopkins University, Baltimore, Maryland 21218, and 7F. M. Kirby Research Center for Functional Brain Imaging, Kennedy Krieger Institute, \\ Baltimore, Maryland 21205
}

Diffusion tensor imaging (DTI) and immunohistochemistry were used to examine axon injury in the rat spinal cord after unilateral $\mathrm{L}_{2}-\mathrm{L}_{4}$ dorsal root axotomy at multiple time points (from $16 \mathrm{~h}$ to $30 \mathrm{~d}$ after surgery). Three days after axotomy, DTI revealed a lesion in the ipsilateral dorsal column extending from the lumbar to the cervical cord. The lesion showed significantly reduced parallel diffusivity and increased perpendicular diffusivity at day 3 compared with the contralateral unlesioned dorsal column. These findings coincided with loss of phosphorylated neurofilaments, accumulation of nonphosphorylated neurofilaments, swollen axons and formation of myelin ovoids, and no clear loss of myelin (stained by Luxol fast blue and 2'-3'-cyclic nucleotide $3 '$-phosphodiesterase). At day 30 , DTI of the lesion continued to show significantly decreased parallel diffusivity. There was a slow but significant increase in perpendicular diffusivity between day 3 and day 30, which correlated with gradual clearance of myelin without further significant changes in neurofilament levels. These results show that parallel diffusivity can detect axon degeneration within $3 \mathrm{~d}$ after injury. The clearance of myelin at later stages may contribute to the late increase in perpendicular diffusivity, whereas the cause of its early increase at day 3 may be related to changes associated with primary axon injury. These data suggest that there is an early imaging signature associated with axon transections that could be used in a variety of neurological disease processes.

\section{Introduction}

Axon injury is present in spinal cord trauma, stroke, and many neurological diseases, such as multiple sclerosis and motor neuron disease (Raff et al., 2002; Medana and Esiri, 2003). It is the likely major underlying substrate of irreversible neurological disability and is now the target of many neuroprotective strategies (Ferguson et al., 1997; Trapp et al., 1998; Bjartmar et al., 1999; Bjartmar and Trapp, 2003). Imaging axon degeneration is important for understanding its mechanisms and developing novel treatments. Among various imaging techniques, diffusion tensor magnetic resonance imaging (DTI) shows great potential. DTI measures the three-dimensional properties of water diffusion, and changes in these measurements may reflect changes in tissue microstructure (Basser et al., 1994; Basser and Pierpaoli, 1996; Le Bihan, 2003; Mori and Zhang, 2006). Studies have shown that measurements of water diffusion anisotropy obtained using DTI, e.g., fractional anisotropy (FA), are sensitive to axon degenera-

\footnotetext{
Received Aug. 18, 2008; revised Jan. 27, 2009; accepted Jan. 30, 2009.

This work was supported by National Multiple Sclerosis Society (NMSS) Collaborative Center Award, NMSS Grant TR-3760-A-3, The Nancy Davis Foundation, and National Institutes of Health Grants EB 003543, ES 012665, NS 059529, and NS 052309.

Correspondence should be addressed to either of the following: Dr. Jiangyang Zhang, Johns Hopkins University School of Medicine, Traylor Building, Room \#208, 720 Rutland Avenue, Baltimore, MD 21205, E-mail: jzhang3@jhmi.edu; or Dr. Peter A. Calabresi, Johns Hopkins Hospital, Pathology Building, Suite 627,600 North Wolfe Street, Baltimore, MD 21287, E-mail: calabresi@jhmi.edu.

D01:10.1523/JNEUROSCI.3941-08.2009

Copyright $\odot 2009$ Society for Neuroscience $\quad$ 0270-6474/09/293160-12\$15.00/0
}

tion in patients (Werring et al., 2000; Pierpaoli et al., 2001; Thomalla et al., 2004; Valsasina et al., 2005; Trip et al., 2006; Agosta et al., 2007). The applicability of DTI in studies of axon degeneration in animal models, both in vivo and ex vivo, has also been demonstrated (Ford et al., 1994; Beaulieu et al., 1996; Fraidakis et al., 1998; Nevo et al., 2001; Stanisz et al., 2001; Schwartz et al., 2005; Kim et al., 2006, 2007; Budde et al., 2007; Mac Donald et al., 2007; Cohen-Adad et al., 2008; Kozlowski et al., 2008; Sun et al., 2008). Several studies suggested that parallel diffusivity (also called axial diffusivity) is sensitive to axon injury, whereas perpendicular diffusivity (also called radial diffusivity) is sensitive to myelin injury (Kim et al., 2007; Sun et al., 2008). Recently, several groups demonstrated that FA, parallel and perpendicular diffusivities are significantly correlated with several histopathological measurements of axon damage distant from the site of inflammatory or traumatic injury in the rodent spinal cord (DeBoy et al., 2007; Budde et al., 2008; Kozlowski et al., 2008). These studies provide a strong rationale for further development of DTI as a surrogate marker of axon degeneration.

This study used high resolution ex vivo DTI to examine Wallerian degeneration (WD) (Waller, 1850) in the rat dorsal root axotomy model (George and Griffin, 1994b). DTI and pathological data at several time points after axotomy were coregistered using nonlinear transformation. The data were used to examine the sensitivity of DTI in detecting pathological events in WD and the spatiotemporal patterns of WD after axotomy. Herein, we show that DTI is exquisitely sensitive to very early spinal cord 
Table 1. Antibodies for pathological characterization of DRG axotomy

\begin{tabular}{|c|c|c|c|c|c|}
\hline Antibody (clone/host) & Pathological feature & Dilution & Supplier & Antigen retrieval & Development \\
\hline CNPase (115-B) & Myelin & $1: 500$ & Millipore Bioscience Research Reagents & $\begin{array}{l}100 \mathrm{~mm} \mathrm{Na} \text { citrate buffer, } \mathrm{pH} 6.0 \text {; } \\
20 \text { min boil }\end{array}$ & $\mathrm{ABC}$ HRP with DAB in PBS, pH 7.4 \\
\hline $\operatorname{APP}(22 C 11)$ & Impaired fast axon transport ${ }^{a}$ & $1: 1000$ & Millipore Bioscience Research Reagents & $\begin{array}{l}100 \mathrm{~mm} \mathrm{Na} \text { citrate buffer, } \mathrm{pH} 6.0 \text {; } \\
20 \mathrm{~min} \text { boil }\end{array}$ & $\begin{array}{l}\text { Alkaline phosphatase-conjugated anti-mouse IgG, } \\
\text { 1:1000; BCIP plus NBT in } 100 \mathrm{~mm} \text { Tris plus } 0.1 \% \\
\text { Tween-20, pH } 9.5\end{array}$ \\
\hline SMI32 (mouse) & Hypophosphorylated neurofilament (abnormal) ${ }^{a}$ & $1: 5000$ & Covance Research Products & $\begin{array}{l}100 \mathrm{~mm} \text { Na citrate buffer, pH 6.0; } \\
20 \text { min boil }\end{array}$ & ABC HRP with DAB in PBS, pH 7.4 \\
\hline SMI31 (mouse) & $\begin{array}{l}\text { Accumulation of hyperphosphorylated neurofilament } \\
\text { (normal) }\end{array}$ & $1: 3000$ & Covance Research Products & $50 \mathrm{~mm}$ Tris, pH 9.5; $20 \mathrm{~min}$ boil & $A B C H R P$ with $D A B$ in PBS, pH 7.4 \\
\hline GFAP (rabbit) & Astrocyte activation $^{a}$ & $1: 1500$ & Sigma & $50 \mathrm{~mm}$ Tris, pH 9.5; $20 \mathrm{~min}$ boil & $\mathrm{ABC}$ HRP with $\mathrm{DAB}$ in $\mathrm{PBS}, \mathrm{pH} 7.4$ \\
\hline Iba-1 (rabbit) & Microglia/macrophage activation ${ }^{a}$ & $1: 1000$ & Wako Bioproducts & $50 \mathrm{~mm}$ Tris, $\mathrm{pH} 9.5 ; 60 \mathrm{~min}$ at $60^{\circ} \mathrm{C}$ & ABC HRP with DAB in PBS, pH 7.4 \\
\hline
\end{tabular}

${ }^{a}$ These antibodies were titrated such that staining for these markers using tissues from unmanipulated animals was very low; pathological processes lead to increases in accumulation (APP and SMI32) or expression (GFAP and Iba-1) of these proteins which these limiting dilutions of antisera can detect.

pathological events $(38-72 \mathrm{~h})$ that occur after dorsal root axotomy.

\section{Materials and Methods \\ Animals}

All experiments and procedures were approved by the Animal Research Committee of the Johns Hopkins University School of Medicine. A total of 28 rats (female Lewis rats, 160-200 g; Charles River Laboratories) were used in this study with four rats for each of the six time points after left $\mathrm{L}_{2}-\mathrm{L}_{4}$ dorsal root axotomy ( $16 \mathrm{~h}, 38 \mathrm{~h}, 3 \mathrm{~d}, 7 \mathrm{~d}, 14 \mathrm{~d}$, and $30 \mathrm{~d}$ after axotomy) and four rats without injury. Dorsal root axotomy was performed on rats deeply anesthetized with isoflurane. Hemilaminectomy at the $\mathrm{L}_{4}-\mathrm{L}_{6}$ vertebral level exposed the cauda equina on the left side, and the left $\mathrm{L}_{2}-\mathrm{L}_{4}$ dorsal roots were transected carefully just proximal to the dorsal root ganglion. The wound was then closed with wound clips, and the animals were allowed to recover. Before ex vivo imaging, the rats were deeply anesthetized and perfused through the heart with cold PBS followed with freshly prepared $4 \%$ paraformaldehyde. To preserve the natural shape and morphology and to avoid damage to the spinal cords or dorsal roots, the cords were kept inside the vertebral columns and were immersed in the same fixative overnight at $4^{\circ} \mathrm{C}$ then transferred to PBS. Three days before imaging, the specimens were transferred to PBS with $1 \mu \mathrm{M}$ Magnevist (gadopentetate dimeglumine; Berlex Imaging) and kept at $4^{\circ} \mathrm{C}$.

\section{Ex vivo DTI and image processing}

Ex vivo imaging of the spinal cord specimens were performed on an 11.7 Tesla spectrometer equipped with a Micro2.5 gradient system (maximum gradient strength 120 Gauss per centimeter; Bruker Biospin). During imaging, the spinal cord specimens, still within vertebral columns, were kept in a magnetic resonance (MR) compatible plastic tube filled with fomblin (Fomblin Perfluoropolyether; Solvay Solexis) to prevent dehydration, and the temperature of the specimens were maintained at $30^{\circ} \mathrm{C}$ via the spectrometer's temperature control system. Images were acquired using a $15 \mathrm{~mm}$ diameter birdcage coil as the radio frequency transmitter and signal receiver and a three-dimensional diffusionweighted multiple spin echo sequence, with a repetition time of $600 \mathrm{~ms}$, echo times (TE) of 27/37/47/57 ms (four spin echoes) and two signal averages. The native imaging resolution was $0.1 \times 0.1 \times 0.1 \mathrm{~mm}$. The matrix size was $320 \times 64 \times 64$, with the readout direction aligned with the long axis of the spinal cord. Images reconstructed from the four spin echoes with $20 \%$ trapezoid appodization window were summed together to form one image. One imaging session includes two images of minimum $b$ value $\left(100 \mathrm{~s} / \mathrm{mm}^{2}\right.$ from the crusher gradients around the refocusing pulse) and six images with maximum $b$ value $\left(1700 \mathrm{~s} / \mathrm{mm}^{2}\right)$ were acquired. The directions of diffusion sensitizing gradients were the same as used in DeBoy et al. (2007). The diffusion time was $15 \mathrm{~ms}$, and the duration of the diffusion gradients was $6 \mathrm{~ms}$. $\mathrm{A} \mathrm{T}_{2}$-weighted image was obtained by averaging the third and forth echo images ( $\mathrm{TE}=47$ and 57 $\mathrm{ms}$, respectively) acquired with the minimum $b$ value. The first and second echo images ( $\mathrm{TE}=27$ and $37 \mathrm{~ms}$, respectively) acquired with the minimum $b$ value have less $\mathrm{T}_{2}$-weighting and were not used. An average diffusion-weighted (aDW) image was obtained by averaging the six diffusion-weighted images with the maximum $b$ value. Because the coil has limited spatial coverage, two separate imaging sessions were neces- sary to cover both the lumbar and thoracic/cervical spinal cord regions. It took $\sim 1.3 \mathrm{~h}$ to acquire each image, and the total imaging time was $\sim 12 \mathrm{~h}$ for each session. The signal-to-noise ratios in the dorsal column white matter measured in the images with the minimum $b$ value was $>20$ for all experiments. The diffusion tensor was calculated using a log-linear fitting method (Basser et al., 1994; Basser and Pierpaoli, 1998). The FA, primary eigenvector $\left(v_{1}\right)$, parallel diffusivity $\left(\lambda_{\|}\right.$, the primary eigenvalue), and perpendicular diffusivity $\left(\lambda_{\perp}\right.$, the average of the secondary and tertiary eigenvalues) were calculated on a voxel-by-voxel basis from diffusion tensor using DTIStudio (http://www.mristudio.org) (Jiang et al., 2006). Fiber tracking was performed with DTI-Studio software using the technique called "fiber assignment by continuous tracking" (Mori et al., 1999; Jiang et al., 2006). Fiber tracking was restricted to voxels with a minimum FA of 0.2 , and the angle of deflection threshold between contiguous voxels was set at $50^{\circ}$.

\section{Immunohistochemistry}

After MR imaging, tissues were dissected out of the bone and cut into 11 cross-sectional pieces 5-7 mm long. These were pre-embedded together in agar-gelatin (Jones and Calabresi, 2007) and then processed for paraffin embedding by routine procedures. Seven-micrometer-thick sections were cut and stained for myelin [Luxol fast blue (LFB), 2' - $3^{\prime}$-cyclic nucleotide $3^{\prime}$-phosphodiesterase], axon markers [SMI-31 for hyperphosphorylated neurofilament, SMI-32 for hypophosphorylated neurofilament, and amyloid precursor protein (APP)], and neuroinflammatory markers (activated microglia or astrocytes as described in Table 1). For immunohistochemistry, SuperFrostPlus-mounted sections were deparaffinized (S3-Histo; BBC Biochemical) and hydrated in graded ethyl alcohols to water, and antigen retrieval was performed as summarized in Table 1. After rapidly cooling slides, endogenous peroxidases were inactivated by immersion in $3 \% \mathrm{H}_{2} \mathrm{O}_{2}$ for 20 min (except for sections to be stained for APP). Nonspecific antibody interactions were blocked with $2 \%$ casein in phosphate buffer supplemented with $0.1 \%$ Tween 20 (except sections to be stained for APP in which 2\% bovine serum albumin dissolved in Tris-buffered saline plus $0.1 \%$ Tween 20 was used), and primary antibodies diluted in the same solution were applied to tissues overnight at $4^{\circ} \mathrm{C}$ in a humid chamber. After washing, biotinylated antibodies were applied at 1:1000 in blocking solution for $45 \mathrm{~min}$ at room temperature (alkaline phosphatase-conjugated anti-mouse IgG was used for APP), followed by the horseradish peroxidase-conjugated avidin biotin complex (Vector Laboratories) (except APP) for $30 \mathrm{~min}$. Alkaline phosphatase (for anti-APP staining) was developed for $1 \mathrm{~h}$ with 5-bromo-4-chloro-3-indolyl phosphate $p$-toluidine salt (BCIP) plus nitro-blue tetrazolium chloride (NBT) in $0.1 \mathrm{M}$ Tris $\mathrm{pH} 9.5$ plus $0.1 \%$ Tween 20 and horseradish peroxidase (HRP) (all other markers) for 5 min with $0.05 \%(\mathrm{w} / \mathrm{v}) 3,3^{\prime}$-diaminobenzidine plus $0.03 \% \mathrm{H}_{2} \mathrm{O}_{2}$ diluted in PBS. APP-immunoreactive slides were mounted with Aquamount, and all others were dehydrated, counterstained with Fast green, cleared in S3-histo and mounted with Vectamount (Vector Laboratories). Images of the dorsal column at approximately $\mathrm{L}_{2}, \mathrm{~T}_{5}$, and $\mathrm{C}_{5}$ were acquired at $10 \times$ using a BX41 microscope equipped with a DP70 camera (Olympus). For LFB staining, sections were deparaffinized in S3 Histo followed by washes in $100 \%$ and $95 \%$ ethanol and immersion in $0.1 \%$ LFB in $95 \%$ ethanol and $0.05 \%$ acetic acid overnight at $60^{\circ} \mathrm{C}$. After completely cool- 
ing to room temperature, slides were rinsed in $95 \%$ ethanol and then destained in multiple rounds of 45 s successive washes in water, $0.05 \%$ lithium carbonate, and $70 \%$ ethanol, and returning to water until contrast between white and gray matter was maximized. LFB-stained sections were then counterstained in aqueous acetate-buffered eosinY $(\mathrm{pH}$ $4.9,0.1 \%)$. For toluidine blue staining, the spinal cords were further fixed in $4 \%$ paraformaldehyde/2\% glutaraldehyde for $2 \mathrm{~h}$, postfixed in OsO4, and embedded in Epon-Araldite resin. Cross sections ( $1 \mu \mathrm{m}$ thick) were stained with toluidine blue for axon/myelin morphology and examined by light microscopy with a $100 \times$ oil-immersion objective lens.

\section{Coregistration of histology and MRI data and quantitative analysis}

Linear normalization. MR images from all animals were first rigidly aligned to a template to facilitate further analysis. Lumbar spinal cord images of an uninjured animal were selected as the template. Using the Landmarker software (http://www.mristudio.org), 16 landmarks were manually placed in the aDW image at the $\mathrm{L}_{1}-\mathrm{L}_{4}$ dorsal roots and ventral roots entry points. Based on these landmarks, MR images of the lumbar spinal cord of each animal were aligned to the template using rigid transformation (rotation and translation).

Nonlinear normalization. The linearly normalized images were cropped to preserve only the $\mathrm{L}_{1}-\mathrm{L}_{4}$ segments of the spinal cord. In the cropped images, signals from tissue outside the spinal cord were removed by intensity thresholding in the aDW images followed by manual editing. The cropped spinal cord only images were then warped to the similarly processed template image using nonlinear transformations generated by Large Deformation Diffeomorphic Metric Mapping (LDDMM) (Miller et al., 2002). After nonlinear normalization, the FA, parallel diffusivity, and perpendicular diffusivity images from the four animals at each time point were averaged. Supplemental Figure S1, available at www.jneurosci.org as supplemental material, shows the average and SD maps of FA, parallel diffusivity, and perpendicular diffusivity. The intensity values in the SD maps reflect both intrasubject variability and residual mismatch between normalized subject and template images.

Coregistration of MRI and histological images. An axial image at the $\mathrm{L}_{2}$ level that best matched the histological slides of the $\mathrm{L}_{2}$ spinal cord was selected from the template image used for nonlinear normalization. The image was cropped to contain only the dorsal column and served as the target for coregistering histological images. Nonlinear landmark-based registration of histological images to the target was performed using the Landmarker software (http://www.mristudio.org). Twenty-one landmarks were manually placed along the boundary and midline of the dorsal column white matter in the target and each histological slide. Landmark based LDDMM (Joshi and Miller, 2000) was used to warp the histological images to the target. The placement of landmarks is shown in Figure 6 .

Selection of regions of interest. After the coregistration of MRI and histological data, one regions of interest (ROIs) (ROI\#1) was defined by tracing the lesion in the ipsilateral dorsal column in the average parallel diffusivity image at $30 \mathrm{~d}$ at the level corresponding to the histology, and another ROI (ROI\#2) of comparable size and shape was defined on the contralateral side. Because all MR images were nonlinearly normalized to the same template, the delineated ROIs could be consistently applied to all animals, including uninjured spinal cords and spinal cords without apparent lesions. In the coregistered SMI-31-stained histology of one injured spinal cord at $30 \mathrm{~d}$ after axotomy (see Fig. 6), another ROI (ROI\#3) was defined in the ipsilateral dorsal column tracing the region with loss of phosphorylated neurofilament. For the contralateral side, the ROI defined in the MR data (ROI\#2) was used. For each animal at each time point, the mean values of FA, parallel diffusivity, and perpendicular diffusivity were computed in each ROI, and the mean and SD over the four animals at each time point were reported.

Optical density. The optical density (OD) of the SMI-31 and LFB staining in ROI\#2 and ROI\#3 was obtained using ImageJ (National Institute of Health; http://rsbweb.nih.gov/ij/) and normalized to the OD in the dorsal columns of uninjured animals.

\section{Statistics}

Nonparametric one-way ANOVA (Kruskal-Wallis) was first performed to evaluate the overall significance of the differences in FA, diffusivities, and optical densities obtained at multiple time points. Post hoc tests were performed using nonparametric Wilcoxon rank-sum tests with Bonferroni correction. We chose nonparametric tests because the FA and diffusivity measurements were not normally distributed. Pearson correlation coefficients were calculated to determine correlations between optical density and diffusivity measurements. Statistical calculations were performed using Matlab (The MathWorks).

\section{Results \\ DTI evidence of axon degeneration in the lumbar dorsal column after dorsal root axotomy}

A thin lesion in the dorsal column first became visible at $3 \mathrm{~d}$ after axotomy in FA, parallel diffusivity, and perpendicular diffusivity images (Fig. 1, yellow arrows). The lesion was most obvious in the parallel diffusivity images as a $0.1-0.2 \mathrm{~mm}$ wide and $\sim 0.6 \mathrm{~mm}$ long, wedge-shaped, hypointense region. The lesion's location and shape in the parallel diffusivity images showed little change from 3 to $30 \mathrm{~d}$ after axotomy. In the perpendicular diffusivity images, the lesion appeared hyperintense, and the contrast between the lesion and surrounding white matter increased over time. At $30 \mathrm{~d}$ after axotomy, the perpendicular diffusivity image clearly showed a hyperintense lesion, the shape and location of which resembled the lesion in the parallel diffusivity images. No lesion was visible in $\mathrm{T}_{2}$-weighted images at any time point.

In horizontal FA and parallel diffusivity images of the rat spinal cords at 3 and $30 \mathrm{~d}$ after axotomy, the lesion appeared as a continuous thin line in the dorsal column across multiple spinal segments (Fig. $2 \mathrm{~A}$, yellow arrows). In the perpendicular diffusivity images, lesions often appeared fragmented at $3 \mathrm{~d}$ after axotomy but became continuous at $30 \mathrm{~d}$ after axotomy (Fig. $2 \mathrm{~A}$ ). At both 3 and $30 \mathrm{~d}$, streamlines in the ipsilateral dorsal column, created by fiber tracking starting at $\mathrm{L}_{2}$, maintained their trajectories within the lesion region at multiple spinal cord segments, both rostral and caudal to $\mathrm{L}_{2}$, without deviating into neighboring regions. Compared with streamlines passing through the contralateral dorsal column, the regions passed by the ipsilateral streamlines had reduced FA and parallel diffusivity and increased perpendicular diffusivity. Three-dimensional renderings of the diffusion tensors in the dorsal column at 3 and $30 \mathrm{~d}$ after axotomy (Fig. 2C) show that the diffusion tensors in the lesions, although still maintaining their original orientation, became flattened compared with the tensors in uninjured regions. This flattening is resulting from decreased length of the long axis (reduced parallel diffusivity) and increased length of the other two axes (increased perpendicular diffusivity).

\section{Histological evidence of axon degeneration and myelin damage after dorsal root axotomy}

The time course of pathological events in this model, especially the changes in the states of axon and myelin, has been well documented in the literature and was re-examined here using immunohistochemistry (Fig. 3). The immunohistochemical markers used in this study and the targeted pathological events are listed in Table 1. Subtle accumulation of nonphosphorylated neurofilament (labeled by SMI-32) was first detected in the ipsilateral dorsal column at $38 \mathrm{~h}$ after axotomy. The accumulation of SMI-32 stain increased from $38 \mathrm{~h}$ to $3 \mathrm{~d}$. At $3 \mathrm{~d}$ after axotomy, SMI-31- and APP-stained histology showed loss of phosphorylated neurofilament and accumulation of APP in the ipsilateral dorsal column. At $7 \mathrm{~d}$ after axotomy, the intensity of SMI-31 staining in the lesion decreased compared with day 3 , and in some 


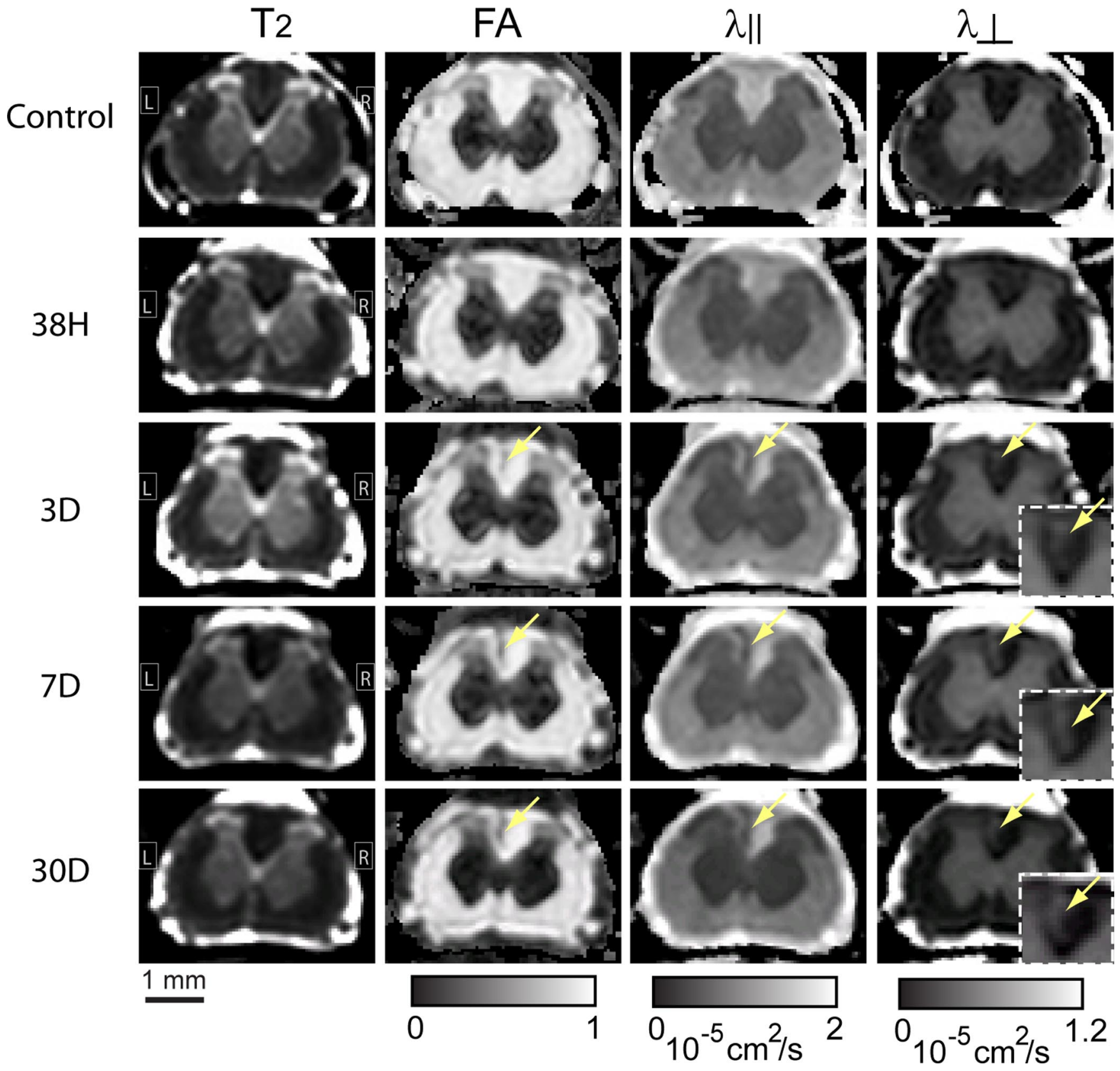

Figure 1. Single-subject axial $\mathrm{T}_{2}$-weighted $\left(\mathrm{T}_{2}\right), \mathrm{FA}$, parallel diffusivity $\left(\lambda_{\|}\right)$, and perpendicular diffusivity $\left(\lambda_{\perp}\right)$ images of $\mathrm{L}_{2}$ spinal cords of uninjured rats (control) and rats $38 \mathrm{~h}(38 \mathrm{H}), 3 \mathrm{~d}(3 \mathrm{D})$, $7 \mathrm{~d}(7 \mathrm{D})$, and $30 \mathrm{~d}(30 \mathrm{D})$ after dorsal root axotomy. The insets in the $\lambda_{\perp}$ image show enlarged dorsal column white matter. Yellow arrows indicate lesions in the dorsal column. $R$, Right; $L$, left (here and in all applicable figures).

cases was barely detectable, whereas SMI-32 and APP staining persisted. The area and location of the lesion remained relatively unchanged from 3 to $30 \mathrm{~d}(\sim 0.4 \mathrm{~mm}$ wide and $1 \mathrm{~mm}$ long). LFB and CNPase staining showed no apparent loss of myelin until 14-30 d (Fig. 3A). GFAP and iba-1 immunohistochemistry (Fig. $3 B$ ) showed little evidence of astrocyte and microglia/macrophage activation. Vacuolation in the lesioned dorsal column appeared at $3 \mathrm{~d}$ after axotomy and became more severe at $30 \mathrm{~d}$ (Fig. $4 A$ ). In toluidine blue-stained sections (Fig. $4 B$ ), myelin ovoids and swollen axons dominated the lesion in the ipsilateral dorsal column at $3 \mathrm{~d}$ after axotomy. Axon density was markedly reduced at $30 \mathrm{~d}$, and the vacated spaces were occupied by astroglia. These observations are in agreement with previous results (George and Griffin, 1994a,b; Buss and Schwab, 2003).
Axon degeneration in the thoracic dorsal column after dorsal root axotomy

Lesions in the thoracic and cervical dorsal column were visible in the parallel diffusivity images at $3 \mathrm{~d}$ after axotomy. They were less apparent in FA and perpendicular diffusivity images (Fig. 5A). At $30 \mathrm{~d}$, the lesions became well defined in the FA, parallel diffusivity, and perpendicular diffusivity images. $\mathrm{T}_{2}-$ weighted images from one of the four animals at the $30 \mathrm{~d}$ time points showed a subtle increase in signal (Fig. $5 A, 30 \mathrm{D} \mathrm{T}_{2}$ ). The lesions in the thoracic dorsal column were closer to the midline of the spinal cord than the lesions in the lumbar dorsal column. The size of the lesion at the level shown in Figure $5 \mathrm{~A}$ was $\sim 0.1 \mathrm{~mm}$ wide (transverse) and $0.6 \mathrm{~mm}$ long (dorsalventral). Histologically, the progression of axon degeneration 
$3 \mathrm{D}$
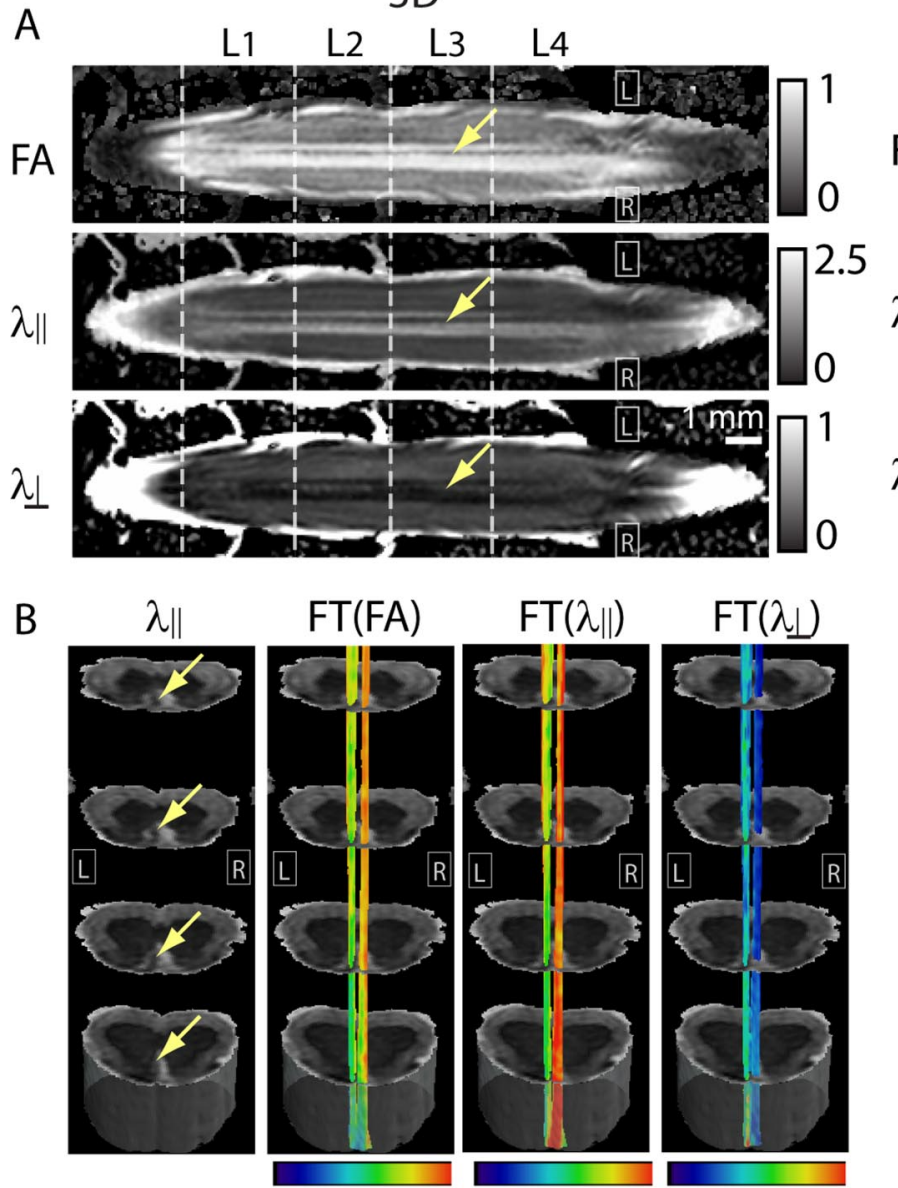

10

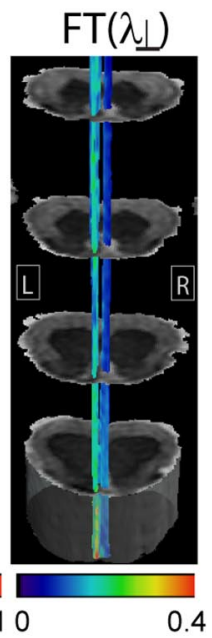

0
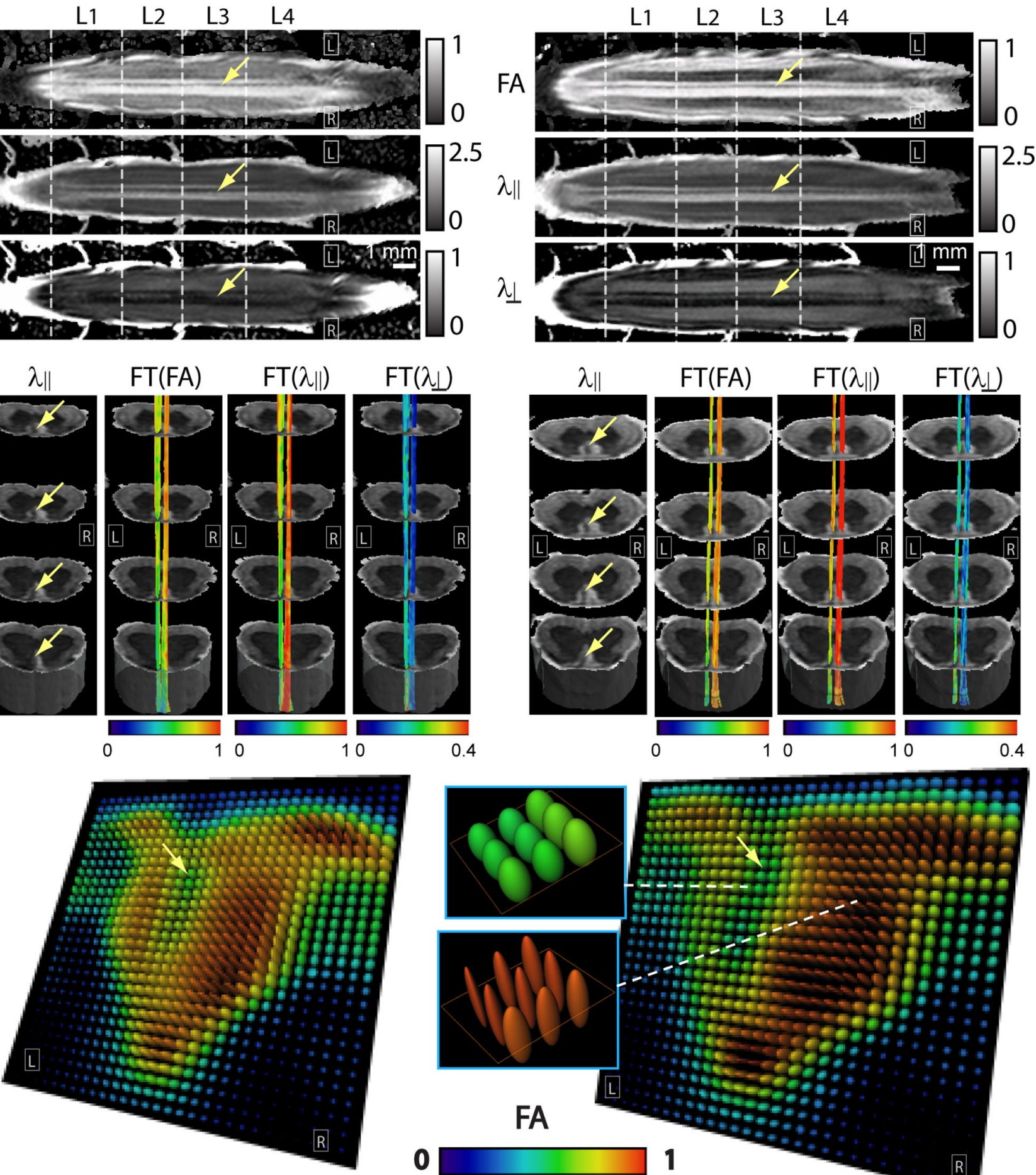

C

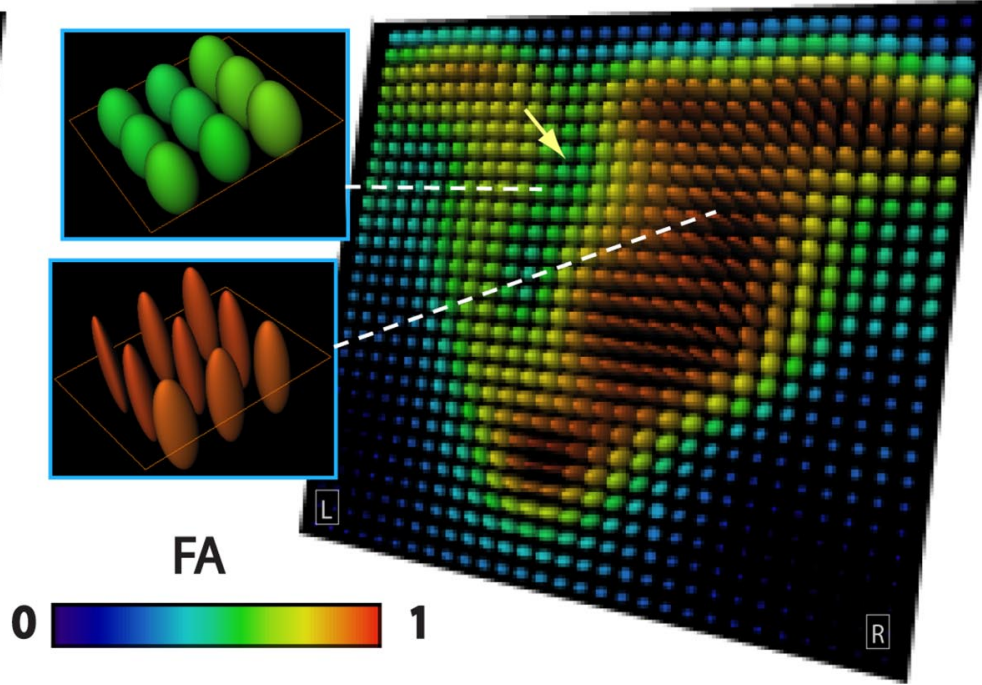

Figure 2. A, Single-subject horizontal images of $F A$, parallel diffusivity $\left(\lambda_{\|}\right)$, and perpendicular diffusivity $\left(\lambda_{\perp}\right)$ showing lesion in the dorsal column at $3 \mathrm{~d}$ (left) and $30 \mathrm{~d}$ (right) after dorsal root axotomy. The locations of the lesion in the dorsal column are indicated by yellow arrows. The unit of the color bars for the parallel and perpendicular diffusivity images is $10^{-5} \mathrm{~cm}^{2} / \mathrm{s}$. $\boldsymbol{B}$, Serial axial parallel diffusivity images at four different levels along the spinal cords at $3 \mathrm{~d}$ (left) and $30 \mathrm{~d}$ (right) after axotomy with streamlines generated by fiber tracking (FT) in the ipsilateral and contralateral dorsal column. Tracking of the ipsilateral streamlines started from seed pixels within the lesion at $L_{2}$, whereas tracking of the contralateral streamlines started from seed pixels in the contralateral dorsal column at $L_{2}$. These streamlines are color coded by the local FA, $\lambda_{\|}$, and $\lambda_{\perp}$ values $\left[\mathrm{FT}(\mathrm{FA}), \mathrm{FT}\left(\lambda_{\|}\right)\right.$, and $\mathrm{FT}\left(\lambda_{\perp}\right)$, respectively] along each streamline. The unit of the color bars for the parallel and perpendicular diffusivity images is $10^{-5} \mathrm{~cm}^{2} / \mathrm{s}$. The axial locations of these selected images are indicated by the dashed lines in the images in $A$. The locations of the lesions are indicated by yellow arrows. In the ipsilateral dorsal column, the streamlines generated by fiber tracking starting at the lesion at $L_{2}$ closely follow the lesion at other levels. $C$, Renderings of diffusion tensors as three-dimensional ellipsoids in the dorsal columns at $3 \mathrm{~d}$ (left) and $30 \mathrm{~d}$ (right) after axotomy. The yellow arrows indicate the locations of the lesions. For each tensor, the lengths of the three axes of each tensor are proportional to the three eigenvalues, and the color reflects the FA value. The two insets in the middle show magnified views of individual diffusion tensors as three-dimensional ellipsoids in the lesion and contralateral dorsal column at $30 \mathrm{~d}$ after axotomy. 


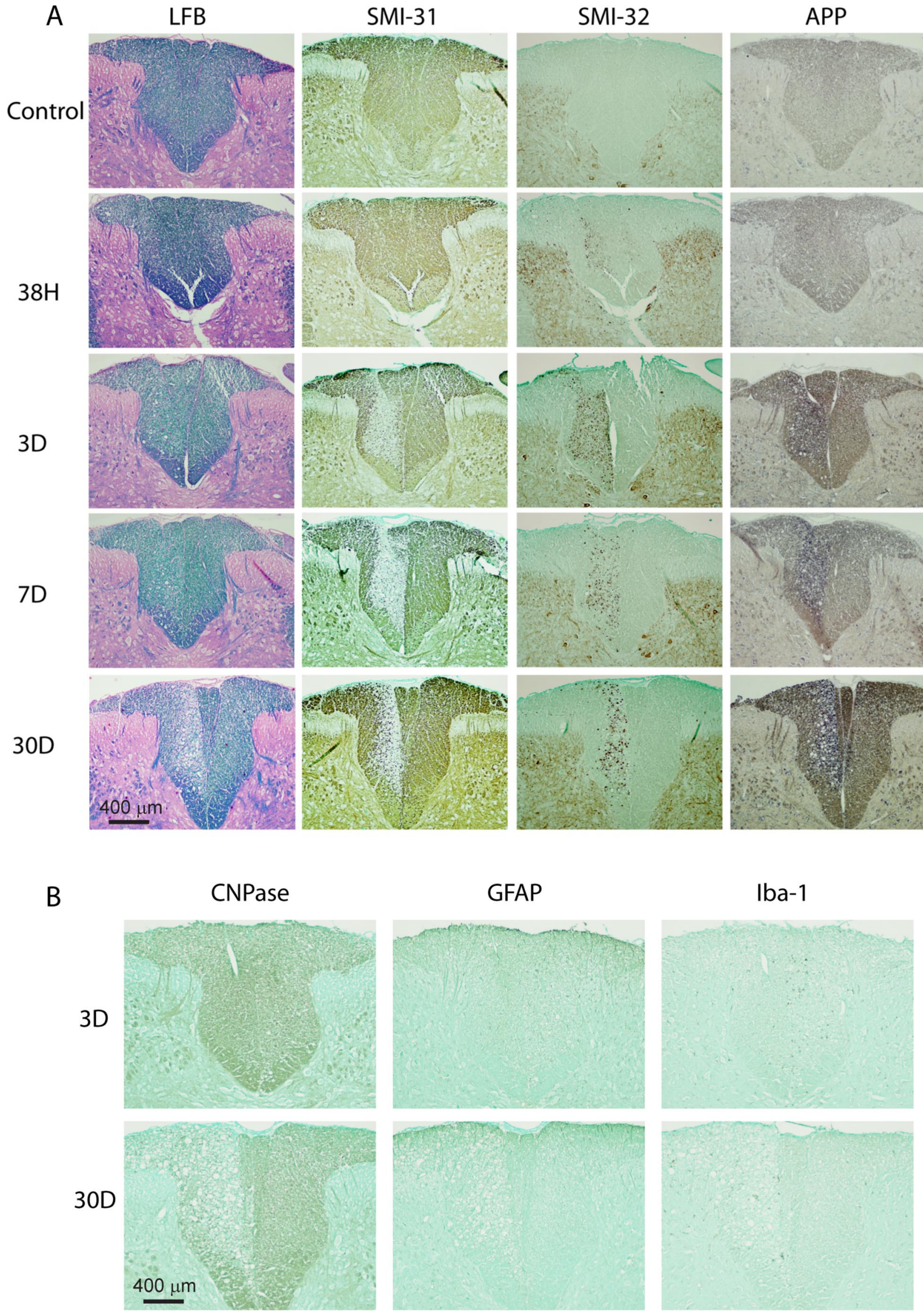

Figure 3. A, LFB-, SMI-31-, SMI-32-, and APP-stained histological images of $\mathrm{L}_{2}$ dorsal column at different time points after axotomy. B, CNPase-, GFAP-, and Iba-1-stained histological images of $L_{2}$ dorsal column at 3 and $30 \mathrm{~d}$ after axotomy. 

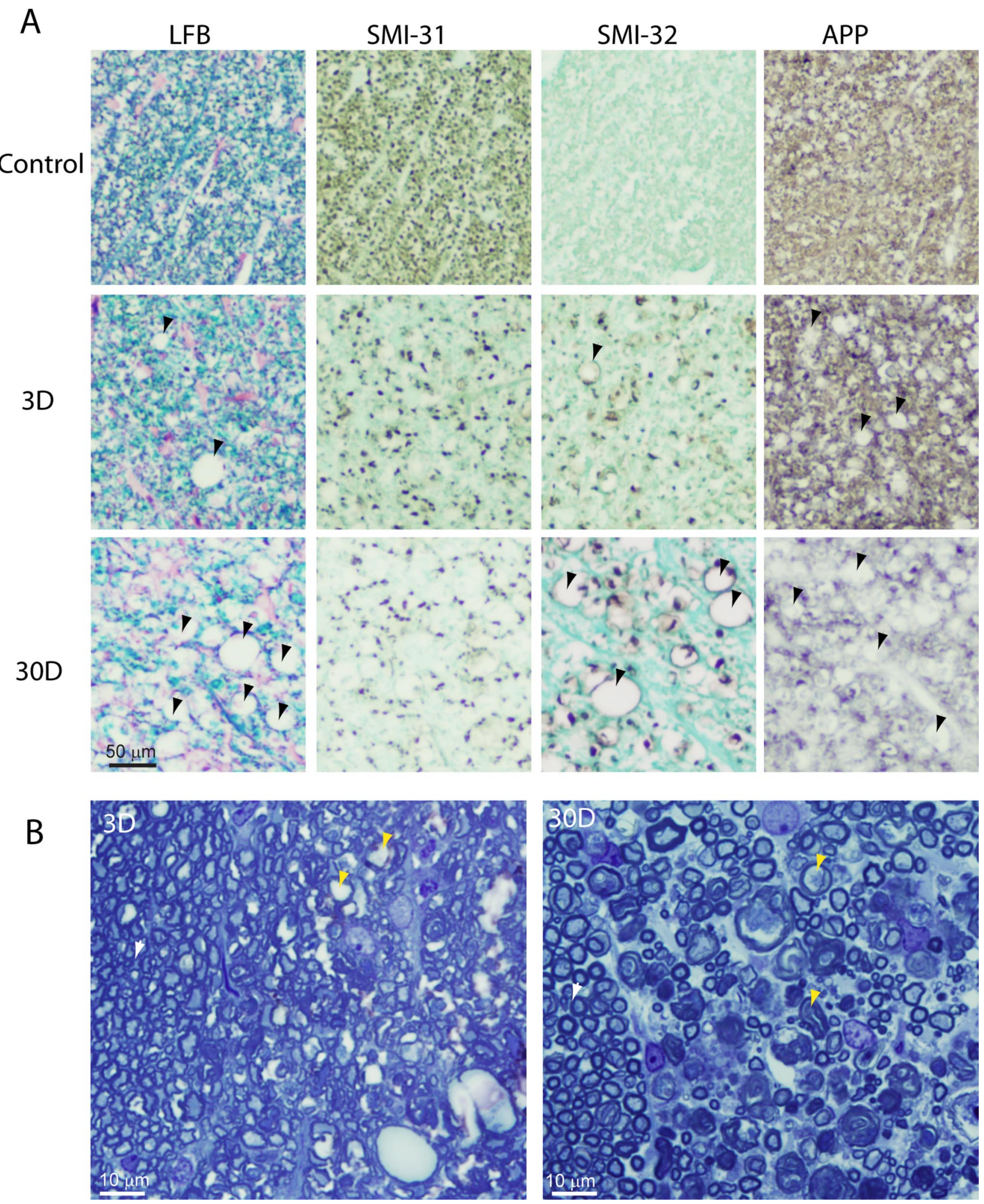

Figure 4. A, LFB-, SMI-31-, SMI-32-, and APP-stained histological images (magnification, $10 \times$ ) show vacuolations (indicated by black arrowheads) in the lesions at $3 \mathrm{~d}(3 \mathrm{D})$ and $30 \mathrm{~d}(30 \mathrm{D})$ after axotomy. No vacuolation is visible in uninjured dorsal column (control). $\boldsymbol{B}$, Toluidine blue-stained histological images (magnification, $100 \times$ ) of the lesion. Yellow arrows indicate the locations of several myelin ovoids in the lesion.

and myelin damage at the thoracic level (Fig. 5B) resembled the progression observed at the lumbar level. The thoracic lesion was limited to the fasciculus gracilis. SMI-31 immunohistochemistry showed that the most dorsal part of the fascic- ulus gracilis contained a small area of uninjured axons (Fig. $5 B$, red arrows) that originated from dorsal roots caudal to the transected $\mathrm{L}_{4}$ dorsal roots. In the parallel diffusivity images, a triangular region that approximated the area of uninjured ax- 

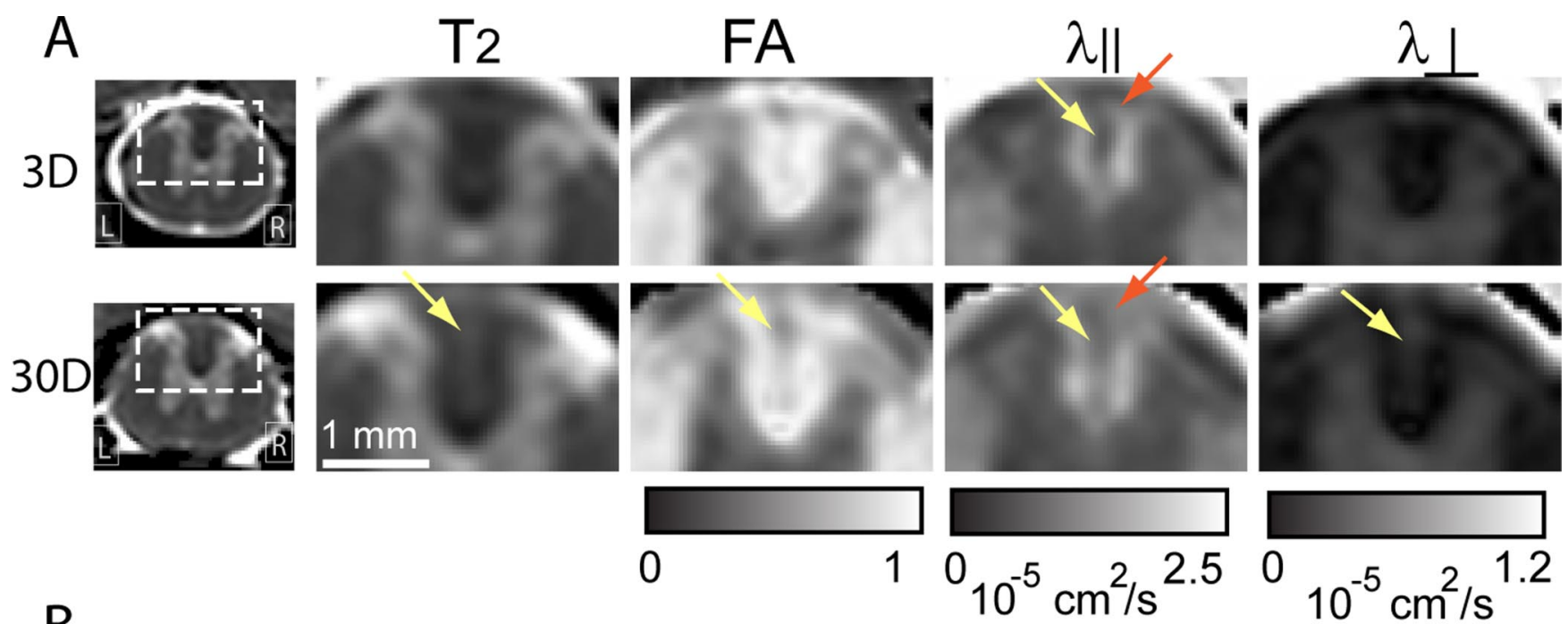

B
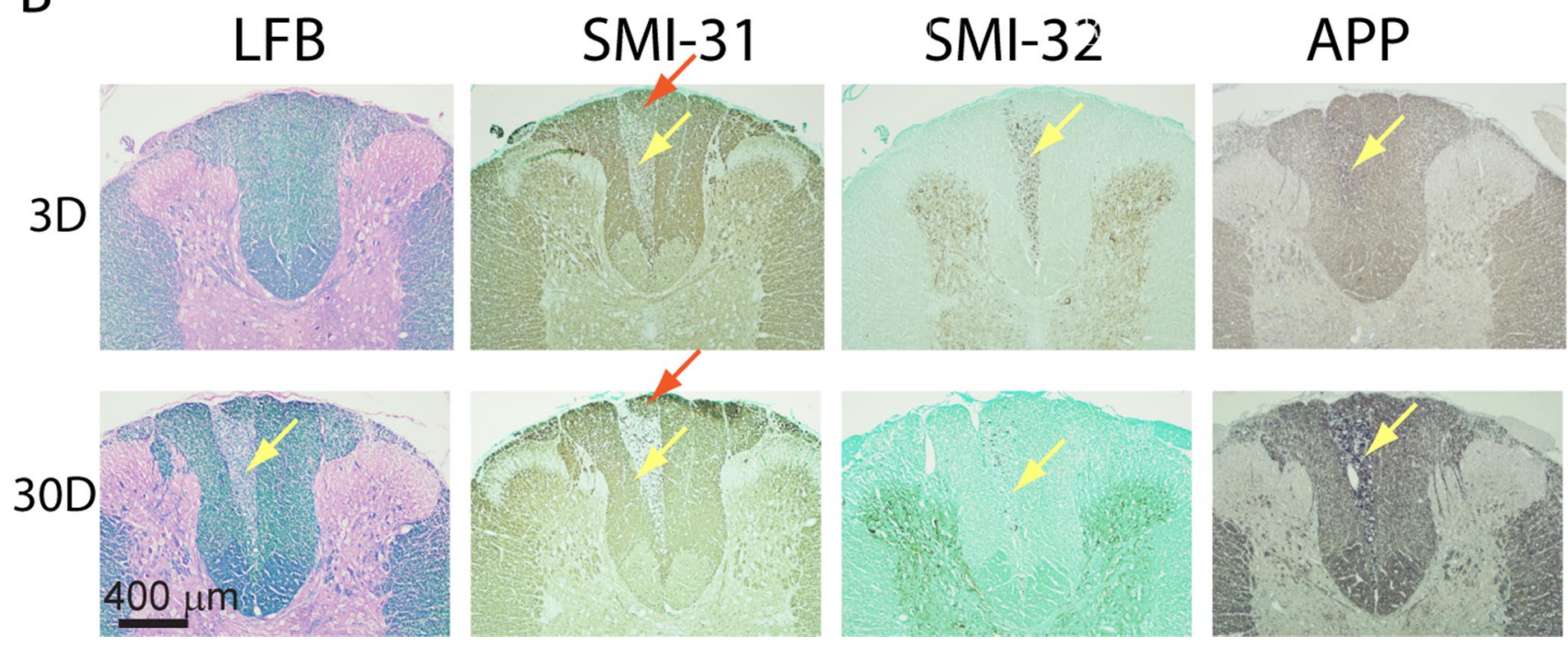

Figure 5. A, Single-subject axial $\mathrm{T}_{2}$-weighted $\left(\mathrm{T}_{2}\right), \mathrm{FA}$, parallel diffusivity $\left(\lambda_{\|}\right)$, and perpendicular diffusivity $\left(\lambda_{\perp}\right)$ images of $\mathrm{T}_{5}$ rat spinal cords at 3 and $30 \mathrm{~d}$ after dorsal root axotomy. Visible lesions are indicated by the yellow arrows. In the $F A, \lambda_{\|}$and $\lambda$ images, only the portion of the dorsal column is shown. In the parallel diffusivity images, a triangular region indicated by the red arrows may contain axons that arise from the dorsal roots caudal to those transected. $B$, Matching LFB-, SMI-31-, SMI-32-, and APP-stained histological images of $\mathrm{T}_{5}$ rat spinal cord dorsal column at 3 and $30 \mathrm{~d}$ after dorsal root axotomy. Red arrows indicate regions that contain axons arise from the uninjured dorsal roots caudal to those transected in this study.

ons in the fasciculus gracilis could be located at a similar anatomical location (Fig. 5A, red arrows).

\section{Quantitative characterization of axon degeneration after dorsal root axotomy}

After normalizing and averaging lumbar MR images to a template (Fig. 6), the lesion could be clearly delineated in the average parallel diffusivity images (indicated by the yellow dashed line, ROI\#1), in which the shape and area of the lesion remained unchanged from 3 to $30 \mathrm{~d}$ after axotomy. To examine the relationship between DTI signals and underlying pathology, histological images were coregistered to the group-averaged MR images of uninjured animals (Fig. 6). In the coregistered SMI-31-stained dorsal column, the lesion was defined as the region with loss of phosphorylated neurofilament (Fig. 6, blue dashed line) (ROI\#3) at $30 \mathrm{~d}$ after axotomy. Figure 6 shows that this region (ROI\#3) contained mostly degenerating axons (as indicated by decreased SMI-31 and increased SMI-32 immunohistochemistry) at $3 \mathrm{~d}$ after axotomy and matched the region with loss of LFB staining at $30 \mathrm{~d}$ after axotomy. Figure 6 shows that the lesion defined in parallel diffusivity images (ROI\#1) was confined within the region defined by loss of SMI-31 immunohistochemistry (ROI\#3).

Figure 7 shows the time courses of FA, parallel diffusivity, and perpendicular diffusivity in the lesion and contralateral dorsal column. The values were obtained from normalized MR data using the regions of interest defined in Figure 6 (ROI\#1 and ROI\#3 correspond to the injured side and are presented in Fig. $7 A, B$, respectively; ROI $\# 2$ corresponds to the contralateral side in both plots.) Similar trends were observed using both methods. Nonparametric one-way ANOVA confirmed significant changes in FA, parallel diffusivity, and perpendicular diffusivity over time. At $3 \mathrm{~d}$ after axotomy, FA and parallel diffusivity in the lesion declined significantly $(p<0.001)$, whereas perpendicular diffusivity in the lesion increased significantly $(p<0.001)$. From 3 to $30 \mathrm{~d}$, FA and parallel diffusivity in the lesion showed no significant change, whereas perpendicular diffusivity in the lesion increased further $(p<0.01)$.

Profiles of FA, parallel diffusivity, and perpendicular diffusivity in the $\mathrm{L}_{1}-\mathrm{L}_{3}$ spinal cord segments at different time points were obtained along the streamlines within the lesion created by fiber 
$\lambda \|$

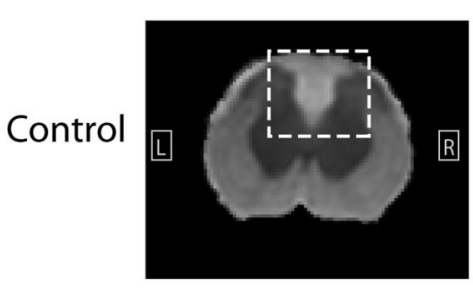

$3 \mathrm{D}$
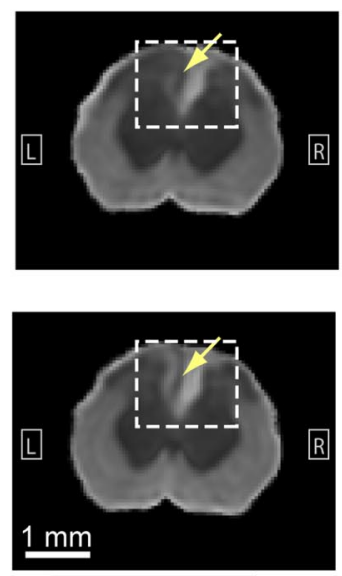

$$
010^{-5} \mathrm{~cm}^{2} / \mathrm{s}^{2.5}
$$
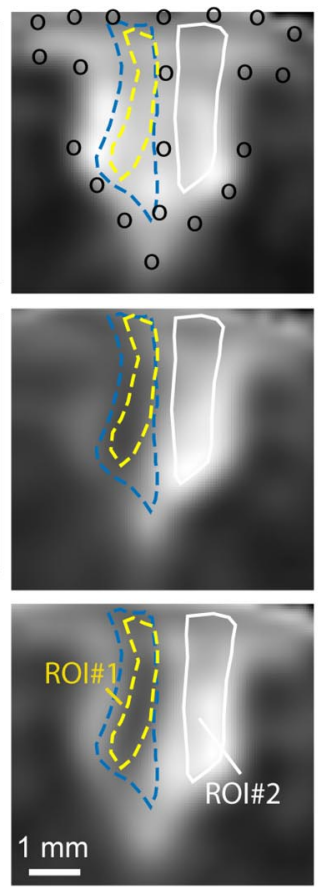

SMI-31

SMI-32
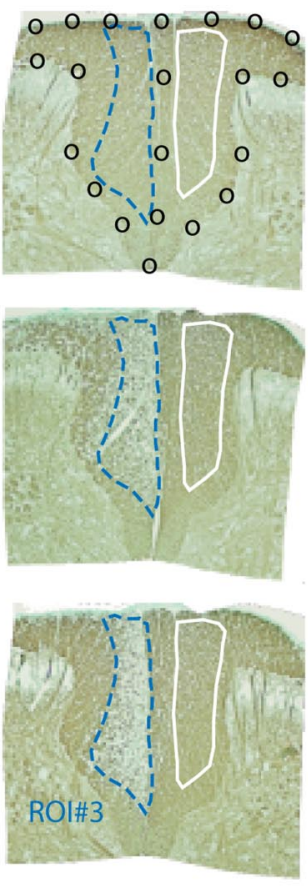
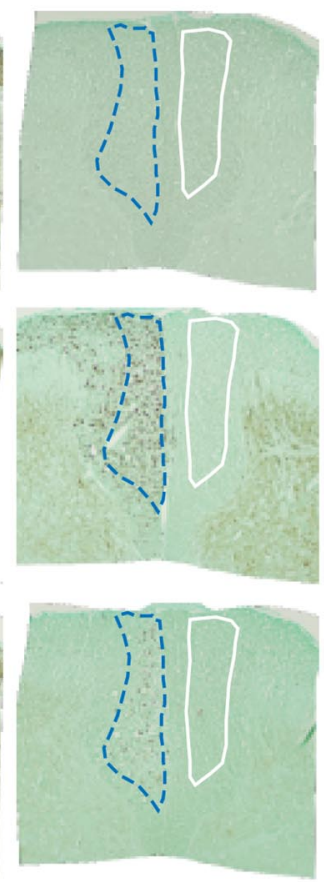

LFB
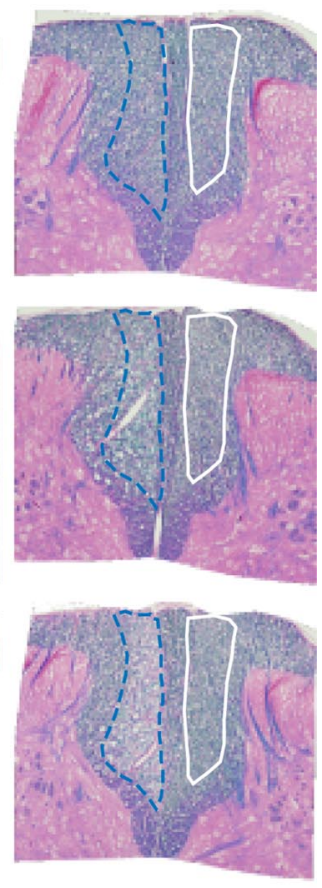

Figure 6. Average parallel diffusivity $\left(\lambda_{\|}\right)$images of $\mathrm{L}_{2}$ rat spinal cords from control animals and animals at 3 and $30 \mathrm{~d}$ after dorsal root axotomy ( $n=4$ for each group) and coregistered $\mathrm{SMI}-31$-, SMI-32-, and LFB-stained histological images. Images in the second column show enlarged dorsal column area in the average $\lambda_{\|}$images. The yellow dashed line outlines the boundary of the lesion as defined in the average $\lambda_{\|}$image at $30 \mathrm{~d}$ after axotomy (ROI\#1). The blue dashed line outlines the region with loss of phosphorylated neurofilament in the SMI-31-stained histology at $30 \mathrm{~d}$ after axotomy (ROl\#3). The white line outlines normal white matter in the contralateral dorsal column (ROl\#2).

tracking. The profiles showed that the changes in FA, parallel diffusivity, and perpendicular diffusivity after axotomy were uniform in the lumbar spinal cord. Optical density measured in SMI-31, and LFB-stained tissue sections showed significant $(p<$ $0.05)$ loss of phosphorylated neurofilament in the dorsal column at $3 \mathrm{~d}$ after injury and significantly reduced myelin at $14 \mathrm{~d}$ after injury, respectively. Significant $(p<0.0001)$ correlations were observed between parallel diffusivity and the OD of SMI-31 ( $r=$ 0.77 ) and between perpendicular diffusivity and the OD of LFB $(r=0.67)$.

\section{Discussion}

This study investigated the sensitivity of DTI to axon degeneration and assessed the spatiotemporal profiles of axon degeneration in the rat dorsal root axotomy model. Previous studies have demonstrated the sensitivity of DTI to axon and myelin injuries in spinal cord inflammatory injury models (Schwartz et al., 2005; Budde et al., 2007, 2008; DeBoy et al., 2007; Cohen-Adad et al., 2008; Kozlowski et al., 2008), in which axon and myelin degeneration happen almost simultaneously because of direct effects of peripheral immune cells as well as activation of microglial cells and astrocytes at the site of degeneration. Unlike inflammatory models, the lesion in this model is free of inflammation and extracellular edema (Fig. 3B) (George and Griffin, 1994a), which provides us a unique opportunity to attribute changes in various MR measurements to particular changes in axon or myelin integrity.

Several previous studies (Beaulieu et al., 1996; Song et al., 2003; Schwartz et al., 2005; DeBoy et al., 2007; Mac Donald et al., 2007; Cohen-Adad et al., 2008; Kozlowski et al., 2008; Sun et al.,
2008) have demonstrated that DTI is sensitive to axon degeneration. In this model, decrease in parallel diffusivity is one of the first imaging signatures associated with axon transection. Examination of the MRI and histological data suggests that the changes in parallel diffusivity in this model closely followed the granular disintegration of the cytoskeleton (GDC), which can be observed in the lumbar dorsal column at $38 \mathrm{~h}$ after axotomy and is complete in most transected axons throughout the entire spinal cord within $3 \mathrm{~d}$ after axotomy (George and Griffin, 1994a). GDC is accompanied by the swelling and collapse of myelin sheath and formation of myelin ovoids (Fig. 4B) (George and Griffin, 1994a). Immunohistochemistry showed accumulation of nonphosphorylated neurofilament in the ipsilateral dorsal column at $38 \mathrm{~h}$ after axotomy followed by loss of phosphorylated neurofilament and accumulation of APP at $3 \mathrm{~d}$ after axotomy at both lumbar (Fig. 3) and thoracic levels (Fig. 5). The decrease in parallel diffusivity was synchronized to these events, and the spatiotemporal pattern observed in the parallel diffusivity image resembled the pattern of GDC. Images of parallel diffusivity revealed a lesion throughout the entire dorsal column at $3 \mathrm{~d}$ after the axotomy. At the $\mathrm{L}_{2}$ level, parallel diffusivity in the lesion dropped $\sim 45 \%$ with respect to contralateral dorsal column and remained stable thereafter. The lesion visualized in the parallel diffusivity images agreed well with the area of significant loss of phosphorylated neurofilament (Fig. 6), and there was a strong correlation between parallel diffusivity and the optical density of SMI-31 immunohistochemistry (i.e., loss of SMI-31 signal) in the lesion (Fig. 7D).

It is not obvious how microstructural changes attributable to 

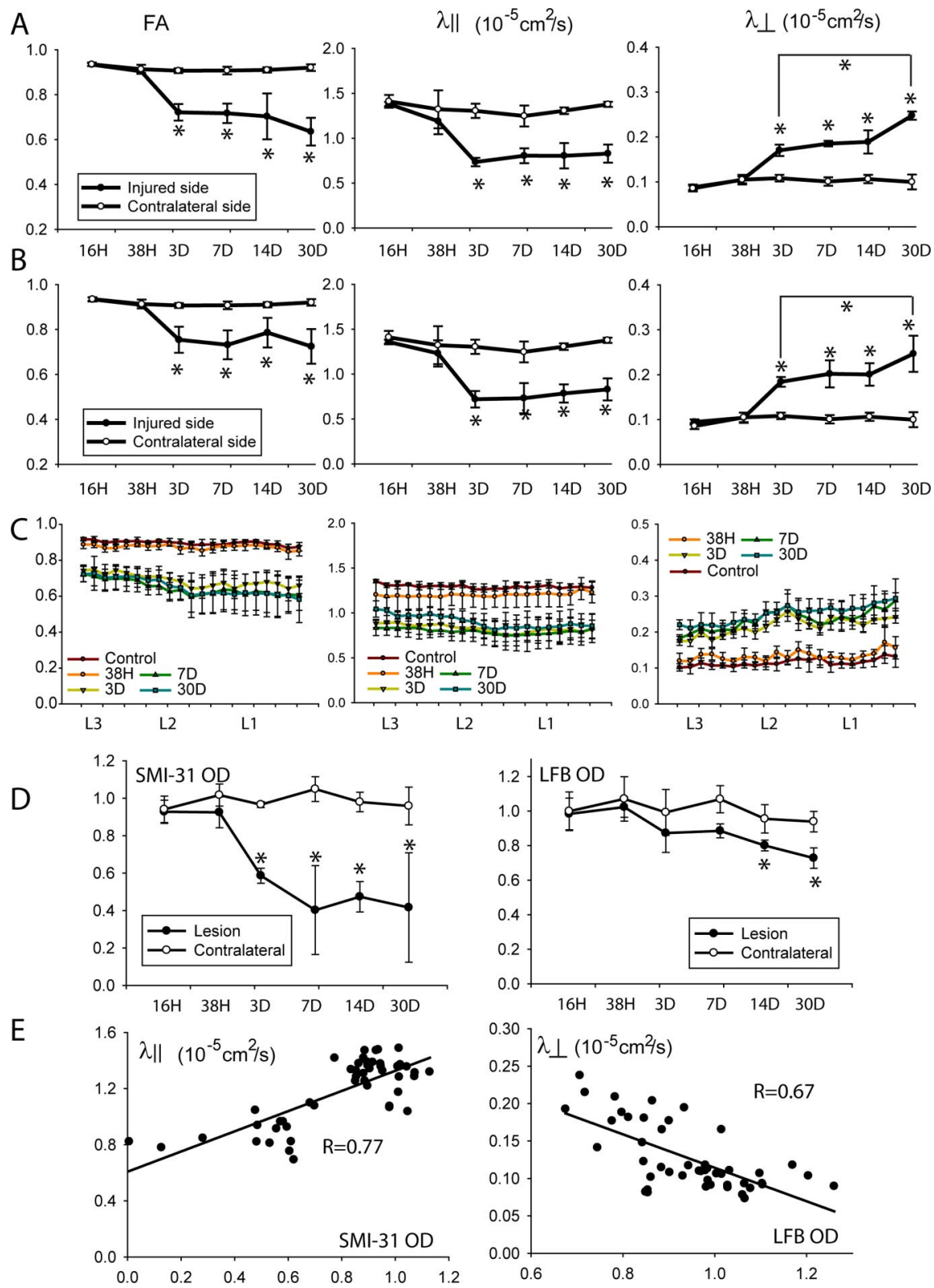

Figure 7. $\quad \boldsymbol{A}, \boldsymbol{B}$, Time courses of $\mathrm{FA}$, parallel diffusivity, and perpendicular diffusivity in the lesion after axotomy from $\mathrm{RO} \# 1(\boldsymbol{A})$ and ROI\#3 (B), and contralateral ROI\#2 (A, B) (Fig. 6) (see Materials and Methods). Note that the horizontal axis uses log scale. *Significant difference exists between control and injured animals ( $p<0.01$ after correction for multiple comparisons). $C$, Profiles of lesion $F A$, parallel diffusivity, and perpendicular diffusivity in $\mathrm{L}_{1}-\mathrm{L}_{3}$ spinal cord obtained from fiber tracking results. $\boldsymbol{D}$, Optical density of SMI-31 and LFB in the lesion (ROI\#3) and contralateral (ROI\#2) white matter. $\boldsymbol{E}$, Correlations between parallel diffusivity and optic density of SMI-31 and correlation between perpendicular diffusivity and optical density of LFB in the lesion (ROI\#3) and contralateral white matter (ROI\#2).

axon degeneration result in a decrease in parallel diffusivity. Beirowski et al. (2005) showed that axons initially break down into small fragments at $37 \mathrm{~h}$ after transection and become fully fragmented with severe vacuolation at $40 \mathrm{~h}$ after transection. The collapsed axon debris and myelin ovoids may hinder water molecule diffusion along injured axons and therefore cause the decrease in parallel diffusivity. The prolonged accumulation of nonphosphorylated neurofilament and APP in the lesion indicates the continuing presence of axon debris from 3 to $30 \mathrm{~d}$ after axotomy. The slow clearance of axon and myelin debris in this model may account for the sustained level of reduced parallel diffusivity between 3 and $30 \mathrm{~d}$ after axotomy. Data generated from other injury models support this hypothesis. For example, in a similar model with slow myelin clearance, Sun et al. (2008) reported prolonged decrease in parallel diffusivity in the mouse optic nerve after transient retinal ischemia (50\% decrease starting at $3 \mathrm{~d}$ after ischemia). When clearance of myelin and axon debris is fast, as in traumatic brain injury (Mac Donald et al., 2007) and in the crushed or transected rat sciatic nerves (Stanisz et al., 2001), parallel diffusivity either showed no decrease or a brief decrease before returning to the normal level. In addition, recent studies showed that parallel diffusivity failed to detect injury in unmyelinated white matter (Drobyshevsky et al., 2007; Ludeman et al., 2008), further suggesting that myelin debris may play a more important role than axon debris in the decrease of parallel diffusivity immediately after GDC. The existence of myelin may be a necessary condition for using parallel diffusivity to reliably detect axon degeneration.

Increase in perpendicular diffusivity is another early imaging signature associated with axon transection in this model. Perpendicular diffusivity in the lesion showed a significant increase at $3 \mathrm{~d}$ after axotomy. Previous studies have demonstrated that myelin damage can result in an increase in perpendicular diffusivity in myelinated white matter (Gulani et al., 2001; Song et al., 2002; Sun et al., 2006a, 2008; Kim et al., 2007; Mac Donald et al., 2007). Several recent studies, however, showed an increase in perpendicular diffusivity in unmyelinated white matter after injury (Drobyshevsky et al., 2007; Ludeman et al., 2008). In this study, LFB staining and CNPase immunohistochemistry indicated no significant loss of myelin in the lesion area (Figs. 3, 7C) at $3 \mathrm{~d}$ after axotomy, whereas toluidine blue staining shows swollen axons and formation of myelin ovoids at the same time point (Fig. $4 B$ ). These reports suggest that primary demyelination is unlikely to be the only factor that contributes to the increase in perpendicular diffusivity. Possible alternatives include the breakdown of the axolemma or a change in its permeability, loss of neurofilaments and microtubules, axon swelling, and an increase in the extracellular space (between myelin and axon membrane) attributable to the collapse of axolemma.

Complete clearance of axon and myelin debris in the spinal cord after axotomy takes more than $90 \mathrm{~d}$ (George and Griffin, 1994a). At $30 \mathrm{~d}$ after axotomy, the lesion showed relatively high diffusion anisotropy $(\mathrm{FA} \sim 0.6)$ (Fig. $7 A, B)$, which indicates that the remaining axon and myelin structural network in the lesion was still sufficient to maintain anisotropic water diffusion. LFB staining (Fig. 3, LFB) showed slow clearance of myelin debris in the ipsilateral dorsal column. Perpendicular diffusivity in the lesion increased significantly from 3 to $30 \mathrm{~d}$ after axotomy (50\%), coinciding with the disintegration of the myelin sheath and clear- 
ance of myelin and axon debris. This increase in perpendicular diffusivity correlated with a decrease in the optical density of the LFB-stained area. These results suggest that perpendicular diffusivity is sensitive to the gradual clearance of myelin.

Although DTI is sensitive to axon degeneration, several inherent limitations can hinder or complicate its use. DTI assumes that tissue water diffusion can be relatively well characterized by a Gaussian model, which may not be valid for certain white matter structures, e.g., structures that contain crossing or branching fibers, or diffusion-weighted imaging experiments at high $b$ values $\left(b>3000 \mathrm{~s} / \mathrm{mm}^{2}\right)$. To obtain more information on tissue microstructure, more complex models and experiments are needed (Assaf and Cohen, 1998; Mulkern et al., 2000; Inglis et al., 2001; Biton et al., 2006). As a result, interpretation of DTI signal changes requires thorough understanding of the underlying pathology and careful validation. Furthermore, the inherent long acquisition time of DTI and the low signal-to-noise ratio in diffusion-weighted images make high resolution in vivo DTI a challenging task. One limitation of the current study is that DTI was performed ex vivo. A previous report demonstrated that parallel and parallel diffusivities measured ex vivo were reduced compared with values measured in vivo, and the sensitivity of parallel diffusivity to axon injury was reduced when measured in ex vivo formalin-fixed tissue specimen (Sun et al., 2006b). Therefore, it is important to develop fast high resolution in vivo imaging techniques for monitoring the spatiotemporal progression of $\mathrm{WD}$. One promising technique is the echo planar imaging (EPI)based acquisition sequences, which can dramatically reduce imaging time. The feasibility of performing high resolution in vivo DTI of rodents using diffusion-weighted EPI sequences has been demonstrated recently (Madi et al., 2005; Asanuma et al., 2008).

In summary, this report demonstrates that DTI is sensitive to very early axon and late myelin degeneration during WD. Parallel and perpendicular diffusivities were able to detect axon degeneration within $3 \mathrm{~d}$ after axons were transected in this model. Although the precise cause of the initial increase in perpendicular diffusivity observed in this study remains to be elucidated, the later increase in perpendicular diffusivity was associated with the gradual clearance of myelin debris. These results suggest that DTI can be a useful tool to monitor axon injury or degeneration in various diseases.

\section{References}

Agosta F, Absinta M, Sormani MP, Ghezzi A, Bertolotto A, Montanari E, Comi G, Filippi M (2007) In vivo assessment of cervical cord damage in MS patients: a longitudinal diffusion tensor MRI study. Brain 130:2211-2219.

Asanuma T, Doblas S, Tesiram YA, Saunders D, Cranford R, Yasui H, Inanami O, Smith N, Floyd RA, Kotake Y, Towner RA (2008) Visualization of the protective ability of a free radical trapping compound against rat C6 and F98 gliomas with diffusion tensor fiber tractography. J Magn Reson Imaging 28:574-587.

Assaf Y, Cohen Y (1998) Non-mono-exponential attenuation of water and $\mathrm{N}$-acetyl aspartate signals due to diffusion in brain tissue. J Magn Reson 131:69-85.

Basser PJ, Pierpaoli C (1996) Microstructural and physiological features of tissues elucidated by quantitative-diffusion-tensor MRI. J Magn Reson B 111:209-219.

Basser PJ, Pierpaoli C (1998) A simplified method to measure the diffusion tensor from seven MR images. Magn Reson Med 39:928-934.

Basser PJ, Mattiello J, LeBihan D (1994) Estimation of the effective selfdiffusion tensor from the NMR spin echo. J Magn Reson B 103:247-254.

Beaulieu C, Does MD, Snyder RE, Allen PS (1996) Changes in water diffusion due to Wallerian degeneration in peripheral nerve. Magn Reson Med 36:627-631.

Beirowski B, Adalbert R, Wagner D, Grumme DS, Addicks K, Ribchester RR,
Coleman MP (2005) The progressive nature of Wallerian degeneration in wild-type and slow Wallerian degeneration (WldS) nerves. BMC Neurosci $6: 6$.

Biton IE, Duncan ID, Cohen Y (2006) High b-value q-space diffusion MRI in myelin-deficient rat spinal cords. Magn Reson Imaging 24:161-166.

Bjartmar C, Trapp BD (2003) Axonal degeneration and progressive neurologic disability in multiple sclerosis. Neurotox Res 5:157-164.

Bjartmar C, Yin X, Trapp BD (1999) Axonal pathology in myelin disorders. J Neurocytol 28:383-395.

Budde MD, Kim JH, Liang HF, Schmidt RE, Russell JH, Cross AH, Song SK (2007) Toward accurate diagnosis of white matter pathology using diffusion tensor imaging. Magn Reson Med 57:688-695.

Budde MD, Kim JH, Liang HF, Russell JH, Cross AH, Song SK (2008) Axonal injury detected by in vivo diffusion tensor imaging correlates with neurological disability in a mouse model of multiple sclerosis. NMR Biomed 21:589-597.

Buss A, Schwab ME (2003) Sequential loss of myelin proteins during Wallerian degeneration in the rat spinal cord. Glia 42:424-432.

Cohen-Adad J, Benali H, Hoge RD, Rossignol S (2008) In vivo DTI of the healthy and injured cat spinal cord at high spatial and angular resolution. Neuroimage 40:685-697.

DeBoy CA, Zhang J, Dike S, Shats I, Jones M, Reich DS, Mori S, Nguyen T, Rothstein B, Miller RH, Griffin JT, Kerr DA, Calabresi PA (2007) High resolution diffusion tensor imaging of axonal damage in focal inflammatory and demyelinating lesions in rat spinal cord. Brain 130:2199-2210.

Drobyshevsky A, Derrick M, Wyrwicz AM, Ji X, Englof I, Ullman LM, Zelaya ME, Northington FJ, Tan S (2007) White matter injury correlates with hypertonia in an animal model of cerebral palsy. J Cereb Blood Flow Metab 27:270-281.

Ferguson B, Matyszak MK, Esiri MM, Perry VH (1997) Axonal damage in acute multiple sclerosis lesions. Brain 120:393-399.

Ford JC, Hackney DB, Alsop DC, Jara H, Joseph PM, Hand CM, Black P (1994) MRI characterization of diffusion coefficients in a rat spinal cord injury model. Magn Reson Med 31:488-494.

Fraidakis M, Klason T, Cheng H, Olson L, Spenger C (1998) Highresolution MRI of intact and transected rat spinal cord. Exp Neurol 153:299-312.

George R, Griffin JW (1994a) Delayed macrophage responses and myelin clearance during Wallerian degeneration in the central nervous system: the dorsal radiculotomy model. Exp Neurol 129:225-236.

George R, Griffin JW (1994b) The proximo-distal spread of axonal degeneration in the dorsal columns of the rat. J Neurocytol 23:657-667.

Gulani V, Webb AG, Duncan ID, Lauterbur PC (2001) Apparent diffusion tensor measurements in myelin-deficient rat spinal cords. Magn Reson Med 45:191-195.

Inglis BA, Bossart EL, Buckley DL, Wirth ED 3rd, Mareci TH (2001) Visualization of neural tissue water compartments using biexponential diffusion tensor MRI. Magn Reson Med 45:580-587.

Jiang H, van Zijl PC, Kim J, Pearlson GD, Mori S (2006) DtiStudio: resource program for diffusion tensor computation and fiber bundle tracking. Comput Methods Programs Biomed 81:106-116.

Jones MV, Calabresi PA (2007) Agar-gelatin for embedding tissues prior to paraffin processing. Biotechniques 42:569-570.

Joshi SC, Miller MI (2000) Landmark matching via large deformation diffeomorphism. IEEE Trans Image Process 9:1357-1370.

Kim JH, Budde MD, Liang HF, Klein RS, Russell JH, Cross AH, Song SK (2006) Detecting axon damage in spinal cord from a mouse model of multiple sclerosis. Neurobiol Dis 21:626-632.

Kim JH, Loy DN, Liang HF, Trinkaus K, Schmidt RE, Song SK (2007) Noninvasive diffusion tensor imaging of evolving white matter pathology in a mouse model of acute spinal cord injury. Magn Reson Med 58:253-260.

Kozlowski P, Raj D, Liu J, Lam C, Yung AC, Tetzlaff W (2008) Characterizing white matter damage in rat spinal cord with quantitative MRI and histology. J Neurotrauma 25:653-676.

Le Bihan D (2003) Looking into the functional architecture of the brain with diffusion MRI. Nat Rev Neurosci 4:469-480.

Ludeman NA, Berman JI, Wu YW, Jeremy RJ, Kornak J, Bartha AI, Barkovich AJ, Ferriero DM, Henry RG, Glenn OA (2008) Diffusion tensor imaging of the pyramidal tracts in infants with motor dysfunction. Neurology 71:1676-1682.

Mac Donald CL, Dikranian K, Bayly P, Holtzman D, Brody D (2007) Diffu- 
sion tensor imaging reliably detects experimental traumatic axonal injury and indicates approximate time of injury. J Neurosci 27:11869-11876.

Madi S, Hasan KM, Narayana PA (2005) Diffusion tensor imaging of in vivo and excised rat spinal cord at $7 \mathrm{~T}$ with an icosahedral encoding scheme. Magn Reson Med 53:118-125.

Medana IM, Esiri MM (2003) Axonal damage: a key predictor of outcome in human CNS diseases. Brain 126:515-530.

Miller MI, Trouve A, Younes L (2002) On the metrics and euler-lagrange equations of computational anatomy. Annu Rev Biomed Eng 4:375-405.

Mori S, Zhang J (2006) Principles of diffusion tensor imaging and its applications to basic neuroscience research. Neuron 51:527-539.

Mori S, Xue R, Crain B, Solaiyappan M, Chacko VP, van Zijl PCM (1999) $3 \mathrm{D}$ reconstruction of axonal fibers from diffusion tensor imaging using fiber assignment by continuous tracking (FACT). Paper presented at the International Society for Magnetic Resonance in Medicine 7th Scientific Meeting, Philadelphia, PA, May.

Mulkern RV, Zengingonul HP, Robertson RL, Bogner P, Zou KH, Gudbjartsson H, Guttmann CR, Holtzman D, Kyriakos W, Jolesz FA, Maier SE (2000) Multi-component apparent diffusion coefficients in human brain: relationship to spin-lattice relaxation. Magn Reson Med 44:292-300.

Nevo U, Hauben E, Yoles E, Agranov E, Akselrod S, Schwartz M, Neeman M (2001) Diffusion anisotropy MRI for quantitative assessment of recovery in injured rat spinal cord. Magn Reson Med 45:1-9.

Pierpaoli C, Barnett A, Pajevic S, Chen R, Penix LR, Virta A, Basser P (2001) Water diffusion changes in Wallerian degeneration and their dependence on white matter architecture. Neuroimage 13:1174-1185.

Raff MC, Whitmore AV, Finn JT (2002) Axonal self-destruction and neurodegeneration. Science 296:868-871.

Schwartz ED, Duda J, Shumsky JS, Cooper ET, Gee J (2005) Spinal cord diffusion tensor imaging and fiber tracking can identify white matter tract disruption and glial scar orientation following lateral funiculotomy. J Neurotrauma 22:1388-1398.

Song SK, Sun SW, Ramsbottom MJ, Chang C, Russell J, Cross AH (2002) Dysmyelination revealed through MRI as increased radial (but unchanged axial) diffusion of water. Neuroimage 17:1429-1436.
Song SK, Sun SW, Ju WK, Lin SJ, Cross AH, Neufeld AH (2003) Diffusion tensor imaging detects and differentiates axon and myelin degeneration in mouse optic nerve after retinal ischemia. Neuroimage 20:1714-1722.

Stanisz GJ, Midha R, Munro CA, Henkelman RM (2001) MR properties of rat sciatic nerve following trauma. Magn Reson Med 45:415-420.

Sun SW, Liang HF, Trinkaus K, Cross AH, Armstrong RC, Song SK (2006a) Noninvasive detection of cuprizone induced axonal damage and demyelination in the mouse corpus callosum. Magn Reson Med 55:302-308.

Sun SW, Liang HF, Le TQ, Armstrong RC, Cross AH, Song SK (2006b) Differential sensitivity of in vivo and ex vivo diffusion tensor imaging to evolving optic nerve injury in mice with retinal ischemia. Neuroimage 32:1195-1204.

Sun SW, Liang HF, Cross AH, Song SK (2008) Evolving Wallerian degeneration after transient retinal ischemia in mice characterized by diffusion tensor imaging. Neuroimage 40:1-10.

Thomalla G, Glauche V, Koch MA, Beaulieu C, Weiller C, Röther J (2004) Diffusion tensor imaging detects early Wallerian degeneration of the pyramidal tract after ischemic stroke. Neuroimage 22:1767-1774.

Trapp BD, Peterson J, Ransohoff RM, Rudick R, Mörk S, Bö L (1998) Axonal transection in the lesions of multiple sclerosis. N Engl J Med 338:278-285.

Trip SA, Wheeler-Kingshott C, Jones SJ, Li WY, Barker GJ, Thompson AJ Plant GT, Miller DH (2006) Optic nerve diffusion tensor imaging in optic neuritis. Neuroimage 30:498-505.

Valsasina P, Rocca MA, Agosta F, Benedetti B, Horsfield MA, Gallo A, Rovaris M, Comi G, Filippi M (2005) Mean diffusivity and fractional anisotropy histogram analysis of the cervical cord in MS patients. Neuroimage 26:822-828.

Waller A (1850) Experiments on the section of glossopharyngeal and hypoglossal nerves of the frog and observations of the alterations produced thereby in the structure of their primitive fibers. Philos Trans R Soc Lond 140:423-429.

Werring DJ, Toosy AT, Clark CA, Parker GJ, Barker GJ, Miller DH, Thompson AJ (2000) Diffusion tensor imaging can detect and quantify corticospinal tract degeneration after stroke. J Neurol Neurosurg Psychiatry 69: $269-272$. 